\title{
Comparative Evaluation of Microsurgical and Conventional Open Flap Surgical Procedure Outcomes in Patients with Periodontitis - A Histopathological \& Scanning Electron Microscopy Study
}

\author{
Shreya Shetty* and Bebika Thoudam \\ Department of Dentistry Program, Ibn Sina National College of Medical Sciences, Saudi Arabia
}

Received: 㭗 June 23, 2018; Published: 此July 13, 2018

*Corresponding author: Shreya Shetty, Department of Dentistry program, Ibn Sina National College of Medical Sciences, Jeddah, Saudi Arabia

\begin{abstract}
Background \& Objectives: The purpose of this study was to assess and compare the periodontal treatment outcomes in patients with chronic periodontitis using open flap debridement approach by microsurgical and conventional surgical methods. And to assess and compare the healing outcomes in both the approaches using histopathology\&immunohistochemistry also the root surface alterations \& presence of residual calculus in the teeth treated by both the methods using scanning electron microscopy (SEM).

Methods: In vitro study design, 5 patients in whom extraction was indicated in teeth in two or more quadrants owing to severe form of periodontitis were selected, one week following surgery, the concerned teeth were extracted with a margin of healing soft granulation tissue around for assessing contents of the healing soft tissue using histopathology \& immunohistochemistry and presence of residual calculus and loss of tooth substance using Scanning Electron Microscopy (SEM)

Results: Residual calculus was seen in both the groups. H\&E staining showed more hemorrhage in control than test group. Immunohistochemistry revealed staining intensity of smooth muscle action more in test than control group.

Conclusion: The H\&E staining examination revealed more areas of hemorrhage in the conventionally treated groups than in the microsurgical groups, but the quality and quantity of fibroblasts could not be assessed in all the sections in both the groups. On the other hand, immunohistochemistry examination revealed that the intensity of smooth muscle actin staining around the endothelium was more prominent in the microsurgery group than in the conventional group which indicates better healing with the use of microsurgery as compared to conventional surgery. The SEM examination revealed residual calculus in both the microsurgically treated and conventionally treated tooth specimens; but loss of tooth substance was more obvious in the conventionally treated specimens in comparison to the microsurgically treated specimens.
\end{abstract}

Keywords: Microsurgery; Macrosurgery; Open Flap Debridement

Abbreviations: SEM: Scanning Electron Microscopy; EHI: Early wound Healing Index; H\&E: Hematoxylin and Eosin

\section{Introduction}

Periodontitis is one of the most common oral inflammatory infectious diseases \& the leading cause of tooth loss and is characterized by the destruction of tooth-supporting tissues. It is a complex phenomenon comprising of bacterial challenge and host response factors [1]. The goal of periodontal therapy is to provide a dentition that functions in health and comfort for the life of the patient [2]. This result is generally achieved by elimination of the periodontal infection resulting from the subgingival colonization of periodontopathic bacteria $[2,3]$. The mechanical removal of bacterial plaque, calculus and toxic material is an effective means of altering the etiology of periodontal disease. The use of microscopes in dentistry has brought on a major revolution in dental practice.
Microsurgery has been proposed in various fields of dentistry. Its perceived advantages in periodontal surgery relate to the enhanced visual acuity associated with magnification and better soft tissue manipulation. A variety of factors associated with soft tissue manipulation and importantly the ability for primary closure of the flap, play an important role in determining the outcome of the flap surgery [4-6].

Complete removal of calculus is essential for achieving a biologically acceptable tooth surface in the treatment of periodontitis.However, evidence has shown that residual calculus exists not only on teeth treated by scaling alone but also on teeth treated by flap surgery followed by scaling \& root planning. Evidence also strongly suggests that optimization of the surgical approach and control of surgical variables, particularly in relation to flap 
design and management, can improve outcomes. In fact, the ability to access the defect along with minute detailing debridement and sealing the flap from contaminated oral environment seems to be the key requirements of open flap debridement procedures. Thus to improve the ability of clinicians to accomplish these outcomes, the use of a microsurgical approach could be considered. Combining enhanced visual acuity with the use of specifically designed microsurgical instruments could allow for more accurate and atraumatic manipulation of soft and hard tissues, thereby increasing the ability to properly debride the defect and the root surfaces [47]. Microsurgery is a practice that embraces three distinct values. First is the enhancement of motor skills to improve surgical ability. This is evident in the smooth hand movements accomplished with increased precision and reduced tremor. Second is the decreased tissue trauma at the surgical site, which is apparent in the use of small instruments and a reduced surgical field.

Third is the application of microsurgical principles to achieve passive and primary wound closure. 8Several studies have documented the use of microsurgery in various root coverage procedures, interdental papillae preservation techniques and periodontal regeneration procedures in intrabony defects. Till date, no clinical studies have documented the use and possible advantages of operating microsurgical loupes in periodontal flap debridement surgery. Hence, this study attempts to compare the clinical outcomes following open flap debridement with and without magnifying loupes. In addition, the study also intends to evaluate and compare the primary flap closure, healing outcomes and hard tissue surface changes that are loss of tooth substance and presence of residual calculus in both the approaches.

\section{Materials and Methods}

Following approval from the Ethical Committee, Bangalore Institute of Dental Sciences \& Post graduate Research Center, Bangalore, a single blinded randomized controlled clinical trial with a split mouth study design was conducted on patients reporting to the Department of Periodontics. The study included 5 patients with chronic localized or generalized periodontitis with grade III mobile teeth which will go for extraction.Presence of any systemic or debilitating diseases, pregnant or Lactating women, a recent history or presence of any acute or chronic infections, history of any drug intake including antibiotics, analgesics or any other drugs 3 months prior to the study, undergone periodontal therapy in the last 6 months, pan/tobacco/bitternut usersor smokers, physically or mentally challenged were excluded from the study. Patients willing to sign on a written consent form, male \& female patients aged between 25-55 years, true suprabony pockets periodontal pockets having probing pocket depth $\geq 5 \mathrm{~mm}$ in two or more than two teeth and in minimum two or more than two quadrants, number of teeth present $\geq 20$ were included in the study. 5 patients in whom extraction was indicated in teeth in two or more quadrants owing to severe form of periodontitis were selected.

\section{Treatment Procedure}

The teeth in these selected patients were randomly divided into 2 groups (test and control) and open flap debridement done; the spilt mouth design was done with the test quadrant being treated by microsurgical open flap debridement and the control quadrant by conventional open flap debridement. The patients were asked to rinse the mouth with $10 \mathrm{ml}$ of $0.2 \%$ chlorhexidine digluconate solution as a pre-procedural rinse. The surgical field was anesthetized using local anesthetic agent. After administration of local anesthesia, intrasulcular incisions were given with a no.15 blade, and then using a periosteal elevator, full thickness buccal and palatal/ lingual flaps were elevated. Granulation tissue was removed using Gracey curettes to provide full access and visibility to the root surfaces. The flaps were approximated to the original level and secured with 3-0 mersilk sutures.In the other site, open flap debridement with microsurgical approaches was done.

The surgical procedure was performed using 3X optimal magnification dental loupe. After administration of local anesthesia, intrasulcular incisions were given with microsurgical blade, and then using a periosteal elevator, full thickness buccal and palatal/ lingual flaps were elevated. The flaps were approximated to the original level and secured with 5-0 mersilk sutures. One week following this treatment, the concerned teeth were extracted with a margin of healing soft granulation tissue around them and the following parameters were assessed and compared.

Contents of the healing soft tissue using histopathology \& immunohistochemistry.

Presence of residual calculus and loss of tooth substance using ScanningElectron Microscopy (SEM).

\section{Results}

\section{Histopathological Findings}

H\&E Staining Observations

A. Test Group: All 5 specimens collected from the healing granulation tissues under histopathological examination showed hyperplastic, parakeratinized and stratified squamous epithelium. The underlying connective tissue was fibrous along with proliferating blood vessels and chronic inflammatory cells. Areas of hemorrhage were observed, but the quality and quantity of fibroblasts could not be assessed in all the sections (Figure 1A).

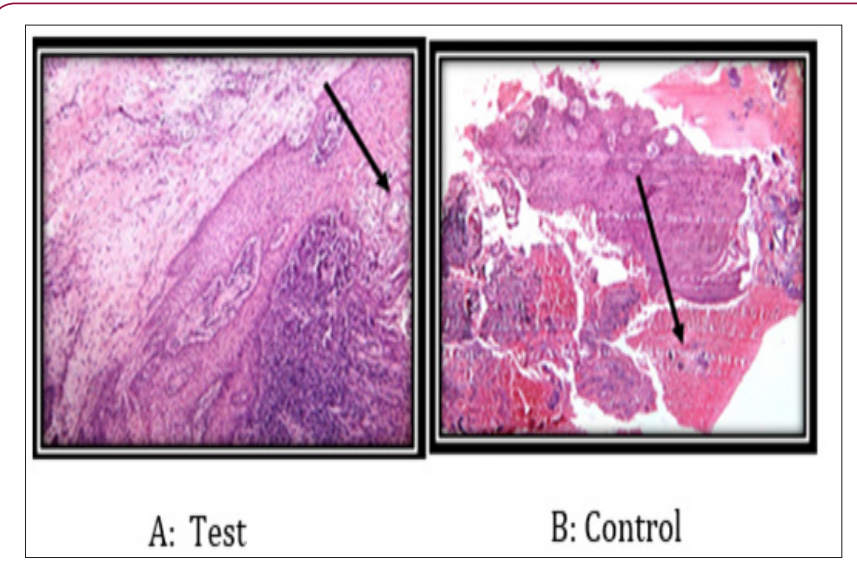

Figure 1: Healing Granulation Tissue - Hematoxylin \& Eosin Staining. 
B. Control Group: All 5 specimens collected from the healing granulation tissues under histopathological examination showed hyperplastic, parakeratinized and stratified squamous epithelium. The underlying connective tissue was fibrous along with proliferating blood vessels and chronic inflammatory cells. Areas of hemorrhage were observed, but the quality and quantity of fibroblasts could not be assessed in all the sections (Figure 1B). There were comparatively more areas of hemorrhage (shown with black arrows) in the control group than in the test group specimens.

\section{Immunohistochemistry Observations}

A. Test Group: Positive staining for smooth muscle actin (shown with red arrows) for vascular endothelium only was observed and no positive staining for myofibroblasts in the collagen fibers was seen (Figure 2A).

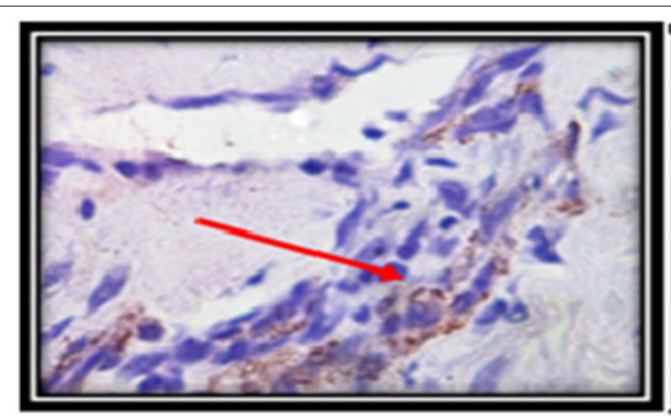

A: Test

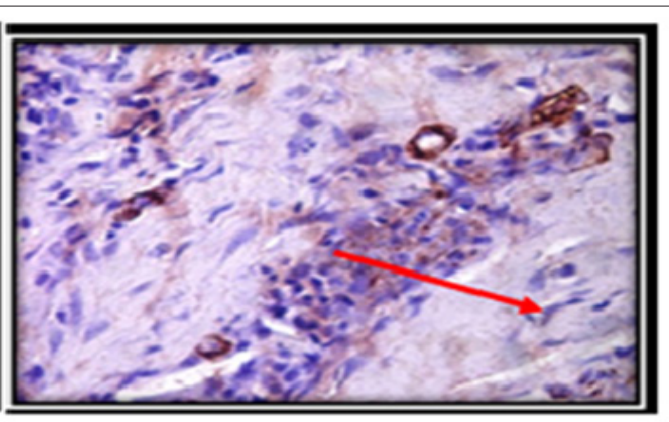

B: Control

Figure 2: Healing Granulation Tissue - Immunohistochemistry.

B. Control Group: Positive staining for smooth muscle actin (shown with red arrows) for vascular endothelium only was observed and no positive staining for myofibroblasts in the collagen fibers was seen (Figure 2B).However, comparatively, the test group specimens showed more intensity of staining of smooth action around endothelial cells as against control group specimens.

\section{Scanning Electron Microscopic Findings}

Residual Calculus: (Circled): Residual calculus was seen in both the test (Figure 3A) and control tooth specimens (Figure 3B).

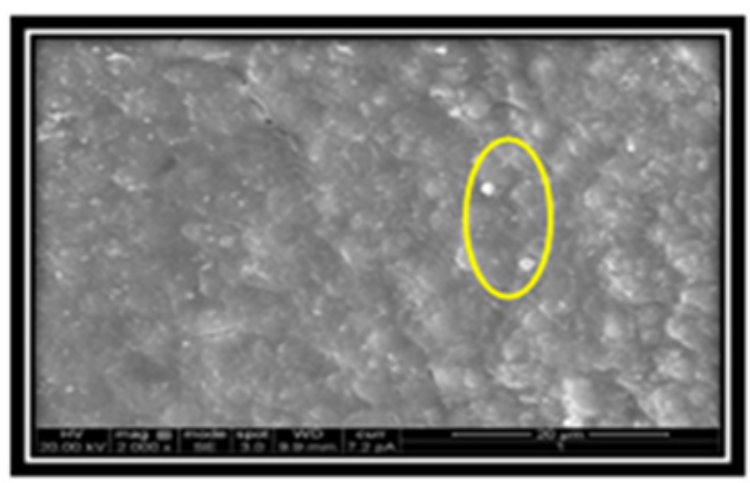

A: Test

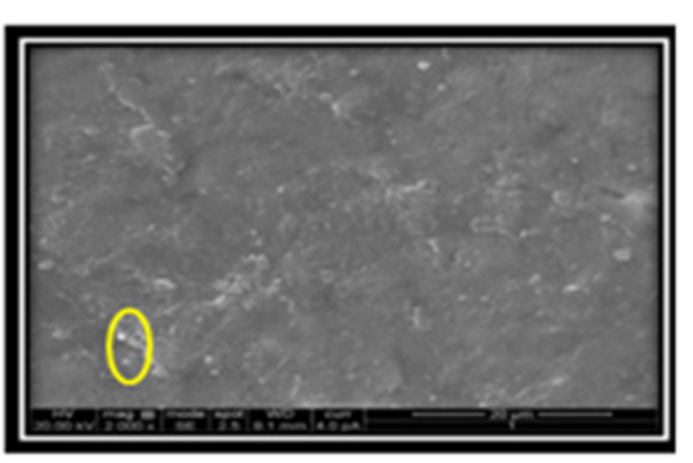

B: Control

Figure 3: Residual Calculus \& Root Surface Changes - Scanning Electron Microscopy.

Loss of Tooth Substance: Tooth specimens of test site showed visible circular mounds of cementum which indicated minimal loss of cementum (tooth substance) during root planning (Figure 3A). On other hand, tooth specimens from the control site showed a smooth surface indicating increased removal of cementum (tooth substance) during root planning (Figure 3B).

\section{Discussion}

Among the various advances in technology available today, periodontal microsurgery is fast gaining popularity. Of prime importance to make any surgical procedure more acceptable are gentle handling of tissues and passive wound closure aiding in primary uneventful healing. So far limited literature exists with regard to using microsurgical technique [5]. patients having teeth with grade III mobility on contralateral sites indicated for extraction were selected and periodontal flap surgeries performed as a split mouth design similar to the clinical study design [7] days following surgery, the concerned teeth were extracted with a margin of healing soft tissue and subjected to SEM and histopathology examination [8]. This study was designed to compare the healing outcomes of microsurgical and microsurgical open flap procedure. A split mouth design was used as this excludes the influence of individual patient 
characteristics and obtains a more powerful estimate of treatment effect with a smaller size [9].

The sample size used in this study was in accordance with the clear majority of clinical periodontal regenerative studies in human [10]. The microsurgical approach involves delicate handling of tissues and precise wound closure which accounts for the favorable early wound healing as reported from studies [11-13]. The sharper and finer surgical blades together with finer suture material used in the microsurgical approach account for the reduced tissue damage along with primary closure of the wound. The improved vascularization after microsurgical approach also enhance the wound healing [12]. And as the healing takes place the inflammatory component reduces thus there were decreased in the distance between the flaps. Other methods of assessing healing clinically include evaluation of color changes of the gingiva; assess patient experiences of the degree of swelling, pain, bleeding and root sensitivity following periodontal surgery [14] by using early wound healing index (EHI) after surgery [11], fluorescent angiograms for the vascularization [12].

This method of evaluating healing has been carried out for the first time and in our opinion is a fairly predictable technique for assessment of primary flap closure assessment. It is completely accepted in literature that effective plaque and calculus removal from the root surface has been a determining factor for the success of periodontal treatment and the control of the disease. Microsurgery uses magnifying glasses, that enhance visual acuity during scaling and root planing and truly is expected to give a better outcome. Lindhe and co-workers $[15,16]$ have suggested that a critical determinant of the success of periodontal therapy is the thoroughness of debridement of the root surface. Studies designed to evaluate the effectiveness of calculus removal after scaling and root planing, with and without surgical intervention, have noted that all calculus is seldom removed from the root surfaces Fleischer et al. [17] reported that regardless of the experience level of the operator, calculusfree roots were obtained more often with surgical access [18] Data has shown that surgical, visual access substantially improves the operator's ability to see and remove calculus in vivo[19]Surfaces treated by scaling and root preparation showed less residual calculus on those treated with surgical access (14\% to $24 \%$ ) than on those treated without surgical access (17\% to $69 \%$ ). In these types of study stereomicroscopy is used to evaluate root surface for residual calculus [19].

Furthermore, research demonstrates that root preparation is enhanced when it is performed under illumination [20]. Till date, no studies exist to indicate whether magnification can enhance the effectiveness of periodontal calculus removal. Because stereomicroscopy is used to evaluate residual calculus on extracted teeth, it seems logical that a surgical operating microscope can enhance the operator's ability to see and remove calculus [20,21] Studies have assessed, loss of tooth substances following hand instrumentation and ultrasonic instrumentation wherein they found tooth substance loss was less in slim line ultrasonic used site [22] Important consideration in periodontal therapy include amount of root surface removed as a result of instrumentation and the roughness of root surface after treatment $[23,24]$. Thus in the present study, it was decided to evaluate the effectiveness of calculus removal from the root surface and assess the presence of residual calculus if any, by SEM in both the test and control sites.

In addition, the root surface alterations following instrumentation were assessed to evaluate whether the test and control sites differ due to variations in accessibility and visibility. Evidence has shown that the best assessment of healing can be done only by histological methods. In the clinical study design, it is not possible to accurately assess and compare the same, hence it was decided to subject the healing granulation tissue obtained from the extracted teeth in both the test and control sites to histologic examination by H\&E stain and immunohistochemistry for better accuracy in comparing the two sites. Animal studies [25] using block specimens including bone, dentin, and surrounding soft tissues were obtained at 10 minutes, 1 and 6 hours, and 1, 3, and 7 days after flap closure and prepared for light and transmission electron microscopic examination of the interface. The 7-day specimens exhibited areas of cell rich connective tissue attachment without inflammatory cells as well as areas showing the fibrin clot in various stages of decomposition.

But the quality and quantity of fibroblasts cannot be assessed by Hematoxylin and eosin (H\&E) staining method. To assess this, immunohistochemistry was done using smooth muscle action as a marker for assessing the activity of fibroblast. Myofibroblasts play an important role in wound healing. Earlier the appearance and more the number of myofibroblast better the quality of wound healing [26]. In parallel studythe hard and soft tissues changes following both the techniques were assessed using SEM and histopathologyimmunochemistry. The hard tissue changes like root surface alterations and residual calculus were assessed using SEM. Residual calculus was found in tooth specimens of both the groups. Till date no studies have been done where residual calculus was assessed using magnification during surgery, but a study by Sherman PR et al. [27] found presence of calculus when observed under a stereomicroscope following ultrasonic scaling and root planing using P-10 universal tip.

Caffesse et al. [28] also found that the percentage of residual calculus was more when scaling and root planing was done alone than with open flap debridement. On the other hand, specimens of microsurgically treated teeth showed less tooth substance loss compared to conventionally treated tooth surfaces. Till date no studies have been reported where tooth substance loss has been assessed in flap debridement done under magnification. However, studies by Ronald K. Hunter et al. [29] and Jotikasthira NE et al. [30] found loss of tooth substance was more in ultrasonic treated teeth than hand scaled teeth when evaluated under the light microscope. In order to ascertain healing more precisely, histologic examination of the healing tissues was done. Comparison between the two groups showed more areas of hemorrhage in the control group than the test group specimens, which is suggestive of more trauma inflicted to the tissues treated by conventional surgery as compared to microsurgery. This further reiterates that microsurgical approach may be less traumatic and more favorable. 
This is in contrast to the study done by Wikesjo et al. [25] wherein they found that 7 days following conventional surgery, areas of complete healing contained a cell rich connective tissue without inflammatory cells, closely adapted to the dentin surface. Other areas demonstrated incomplete healing showing residual fibrin, red blood cells, and polymorphonuclear neutrophils in various stages of decomposition. Myfibroblasts play an important role in wound healing. Earlier the appearance and more the number of myofibroblast better the quality of wound healing [31]. The presence of myofibroblast in the healing tissues is a good indicator of healing. To assess this, immunohistochemistry was done using smooth muscle actin as a marker for assessing the activity of fibroblast.However, in our study, positive staining for myofibroblasts was not observed in either of the groups.

This could be possibly due to the reason that the specimen was taken on the $7^{\text {th }}$ day following surgery and studies have shown that myofibroblasts start appearing from the 8th day and go on increasing between $9^{\text {th }}$ and $12^{\text {th }}$ day during the healing process [32]. On the other hand, positive staining for smooth muscle actin for vascular endothelium was observed in both the microsurgical and conventionally treated specimens. In the conventional group, one out of 5 specimens showed deeper intensity of staining for smooth muscle actin around the vascular endothelium. In comparison, in the microsurgical group 4 out of 5 specimens; showed more intensity of staining of smooth actin around vascular endothelium. Studies have shown that the incidence and severity of complications following periodontal surgery correlate well with the duration of the procedure and the degree of trauma induced at the time of the surgery. This clearly demonstrates that the microsurgery treated sites showed relatively better healing than the conventionally treated sites owing to decreased trauma to the surgical site.

\section{Conclusion}

The following conclusion could be drawn from the present study

a) The SEM examination revealed residual calculus in both the microsurgically treated and conventionally treated tooth specimens; but loss of tooth substance was more obvious in the conventionally treated specimens in comparison to the microsurgically treated specimens.

b) The H\&E staining examination revealed more areas of haemorrhage in the conventionally treated groups than in the microsurgical groups, but the quality and quantity of fibroblasts could not be assessed in all the sections in both the groups. On the other hand, immunohistochemistry examination revealed that the intensity of smooth muscle actin staining around the endothelium was more prominent in the microsurgery group than in the conventional group which indicates better healing with the use of microsurgery as compared to conventional surgery.

\section{Limitations}

a) More sophisticated healing assessment methods like fluorescein angiogram, flow cytometry using markers for various cells and cytokines; and also, immunohistochemistry evaluation of healing using various MMPs as markers could also be done.

b) Larger sample size involving a larger cross section of the population needs to be carried out.

\section{References}

1. Carranza, Newman, Takei, KlokkevoldAdvanced Diagnostic Techniques (10 ${ }^{\text {th }}$ edn.). In: Carranza, Newman, Takei, Klokkevold (Eds.). Clinical Periodontology pp. 582.

2. Grant DA, Stern IB, Listgarten MA (1990) Periodontics the tradition of Gottleib \& Orban ( $6^{\text {th }}$ edn.). In: Grant DA, Stern IB, Listgarten MA (Eds.). by Mosby publications pp. 315-375.

3. McDonell HT, Mills MP Principles and practice of periodontal surgery. In Rose LF, Genco RJ, Cohen DW (Eds.). Periodontics Medicine surgery and implants. Elsevier Mosby publications pp. 358-404.

4. Stephen K Harrel, J Periodontol (1999) A minimally invasive surgical approach for periodontal regeneration: Surgical Technique and observations 70(12): 1547-1557.

5. Tibbetts L, Shanelec DA (1994) An overview of periodontal microsurgery. Curr Opin Periodontol pp. 187-193.

6. Dennis A Shanelec, Leonard S Tibbetts(1996) A perspective on the future of periodontal microsurgery. Periodontology 2000 11: 58-64.

7. Leonard STibbetts, Dennis A Shanelec(1998) Periodontal microsurgery. Dental Clinics of North America 42(2): 339-359.

8. Shanelec DA(2003) Periodontal Microsurgery. J Esthet Restor Dent 15(7): 118-123.

9. Hujoel DP, DeRuen CT(1992) Validity of split mouth trails. J Clin Periodontol 19(9): 625- 627.

10. Antezaks AA, Tulloch JFC, Berkey CS(1990) Split mouth and cross over designs in dental research. J Clin Periodontotol 17(7): 446-453.

11. Wachtel H, Schenk G, Böhm S, Weng D, Zuhr O, et al. (2003) Microsurgical access flap and enamel matrix derivative for the treatment of periodontal intrabony defectsa controlled clinical study. J Clin Periodontol 30(6): 496-504.

12. BurkhardtR,Lang NP (2005) Coverage of localized gingival recessions: comparison of micro- and macrosurgical techniques. J Clin Periodontol 32(3): 287-293.

13. Fickl S, Thalmair T, Kebschull M, Böhm S, Wachtel H (2009) Microsurgical access flap inconjunction with enamel matrix derivative for the treatment of intra-bony defectsa controlled clinical trial. J Clin Periodontol 36(9): 784-790.

14. Curtis JW , McLain JB, Hutchison RA (1985) The incidence and severity of complications and pain following periodontal surgery. J Periodontol 56(10): 597-601.

15. Lindhe J, Nyman S (1984) Long term maintenance of patients treated for advanced periodontal disease. J Clin Periodontol 11(8): 504-514.

16. Lindhe J, Westfelt E, Nyman S, Socransky SS, Haffajee AD (1984) Long term effect of surgicalnonsurgical treatment of periodontal disease. J Clin Periodontol 11(7): 448-458.

17. Buchanan SA, Robertson PB (1987) Calculus removal by scaling/root planing with and without surgical access. J Clin Periodontol 58(3): 159163.

18. Fleischer HC, Mellonig JT, Brayer WK, Gray JL, Barnett JD (1989) Scaling and root planing efficacy in multirooted teeth. J Periodontol 60(7): 402409.

19. Guity M Rabbani, Major MAshf, Raul G Caffesse (1981) The effectiveness of subgingival scaling and Root planing in calculus removal.J Periodontol 53(3): 119-123. 
20. Belcher JM (2001) A perspective on periodontal microsurgery. Int J periodontic Restorative Dent 21(2): 191-196.

21. Osuna T (2003) Magnification use in dental hygiene. Access supplement 6:1-8.

22. Preeti Marda, Shobha Prakash, Ch Devaraj, S vastardis (2012) Comparison of root surface instrumentation using manualultrasonic and rotary instruments. An in vitro study using scanning electron microscopyJ Ind Society Periodontology 23(2): 164-174.

23. Ritz L, Hefti A, Rateitschak KH (1991) An in vitro investigation on the loss of root substance in scaling with various instruments. J Clin Periodontol 18(9): 643-647.

24. Leknes KN, Lie T, Wikesjo U, Bogle GC, Selvig KA (1994) Influence of tooth instrumentation roughness on subgingival microbial colonization. J Periodontol 65(4): 303-308.

25. Wikesjo UME, Crigger M, Niveus R, Selvig KA (1991) Early healing events at the dentin connective tissue interface. Light and transmission electron microscopy observations J Periodontol 62(1): 5-14.

26. DarbyI, Skalli O, Gabbiani G (1990) Alphasmooth muscle actin is transiently expressed by myofibroblasts during experimental wound

ISSN: 2574-1241

DOI: 10.26717/BJSTR.2018.06.001407

Shreya Shetty. Biomed J Sci \& Tech Res

(C) $($ C This work is licensed under Creative

Submission Link: https://biomedres.us/submit-manuscript.php healing. A Journal of Technical Methods and Pathology 63(1): 21-29.

27. Sherman PR, Hutchens LH, Jewson LG (1990) The effectiveness of subgingival scaling and root planing. I Clinical detection of residual calculus J Periodontol 61(1): 9-15.

28. Caffesse RG, Sweeney PL, Smith BA (1986) Scaling and root planing with and without periodontal flap surgery. J Clin Periodontol13(3): 205-210.

29. Hunter RK, Oleary TJ, Kafrawy AH (1984) The Effectiveness of Hand versus Ultrasonic Instrumentation in Open Flap Root Planing. J Periodontol 55(12): 697-703.

30. Jotikasthira NE, Lie T, Leknes KN(1992) Comparative in vitro studies of sonicultrasonic and reciprocating scaling instruments. J Clin Periodontol 19(8): 560-569.

31. Darby I, Skalli O, Gabbiani G (1990) Alphasmooth muscle actin is transiently expressed by myofibroblasts during experimental wound healing. A Journal of Technical Methods and Pathology 63(1): 21-29.

32. Filioreanu AM, Popescu E, Neamtu M, Petreus T, Badescu L, et al. (2010) Immunohistochemical study regarding fibroblast populations involved in tissue regeneration processes. Annals of RSCB 16(1): 160-164.

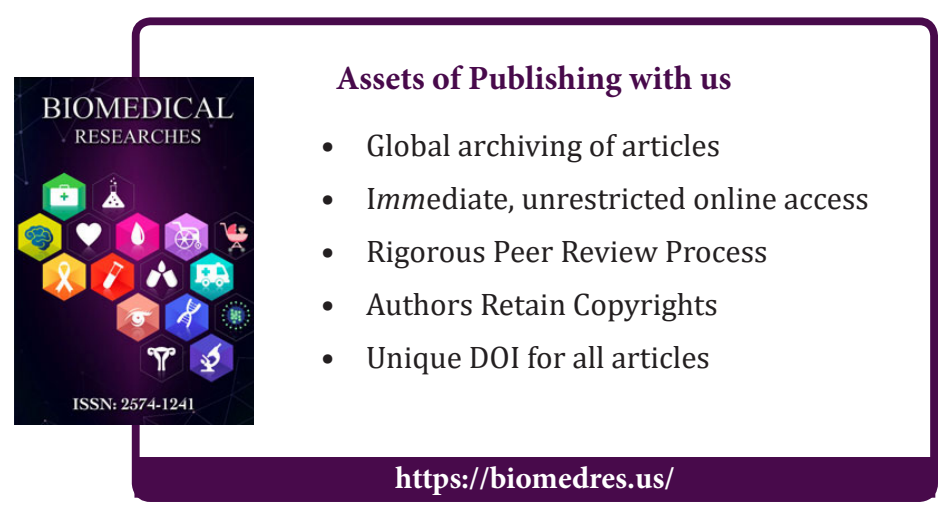

